\title{
Patterning Microsphere Surfaces by Templating Colloidal Crystals
}

\section{Gang Zhang, Dayang Wang* and Helmuth Möhwald}

Max Planck Institute of Colloids and Interfaces, D-14424, Potsdam, Germany

\section{Supporting Information:}

\section{Experimental details:}

$10 \mathrm{wt} \%$ aqueous suspensions of polystyrene of 270 and $925 \mathrm{~nm}$ in diameter and silica spheres of 300 and $520 \mathrm{~nm}$ in diameter were purchased from Microparticles GmbH, Germany. These suspensions were diluted to1.0-3.0 wt\%.

Both silicon wafers and glass slides, cleaned with piranha solution $\left(\mathrm{H}_{2} \mathrm{SO}_{4}: \mathrm{H}_{2} \mathrm{O}_{2}\right.$ $=3: 1$, were used as substrates for colloidal crystallization. The colloidal crystals were prepared by a dipping process; the dipping speeds were controlled in the range of 0.5-12 $\mu \mathrm{m} / \mathrm{s}$. The thickness of the resulting colloidal crystals was tuned by optimizing the concentrations of the colloidal sphere suspensions and dipping speeds.

By varying Au evaporation time, $3.2 \mathrm{~nm}$ thick $\mathrm{Cr}$ and $30 \mathrm{~nm}$ or $60 \mathrm{~nm}$ thick $\mathrm{Au}$ films were deposited within the as-made colloidal crystals in high vacuum. During $\mathrm{Au}$ vapor deposition, no distortion of colloidal spheres and crystal structure were observed. After Au deposition, we placed double sided adhesive tapes on the Aucoated surfaces of colloidal crystals and subsequently covered glass slides atop adhesive tapes. We press glass slides by $0.2 \mathrm{~kg} / \mathrm{cm}^{2}$ for $10 \mathrm{~min}$ in order to ensure that glass slides and colloidal crystal surfaces were closely stocked on adhesive tapes. Along with peeling off glass slides, adhesive tapes and the top monolayers of colloidal spheres were stripped off, leaving behind Au-pattern on colloidal spheres in the

\footnotetext{
* To whom the correspondence should be addressed. Fax: +49 331567 9202. Email: dayang@mpikggolm.mpg.de
} 
second layers. Following the same procedure, we may peel off colloidal spheres layer by layer.

SEM images were recorded with a Gemini LEO 1550 instrument operated at $3 \mathrm{kV}$. In our experiments in order to directly reveal the Au patterns on colloidal spheres, the samples without Au sputter-coating were used for SEM measurements. 


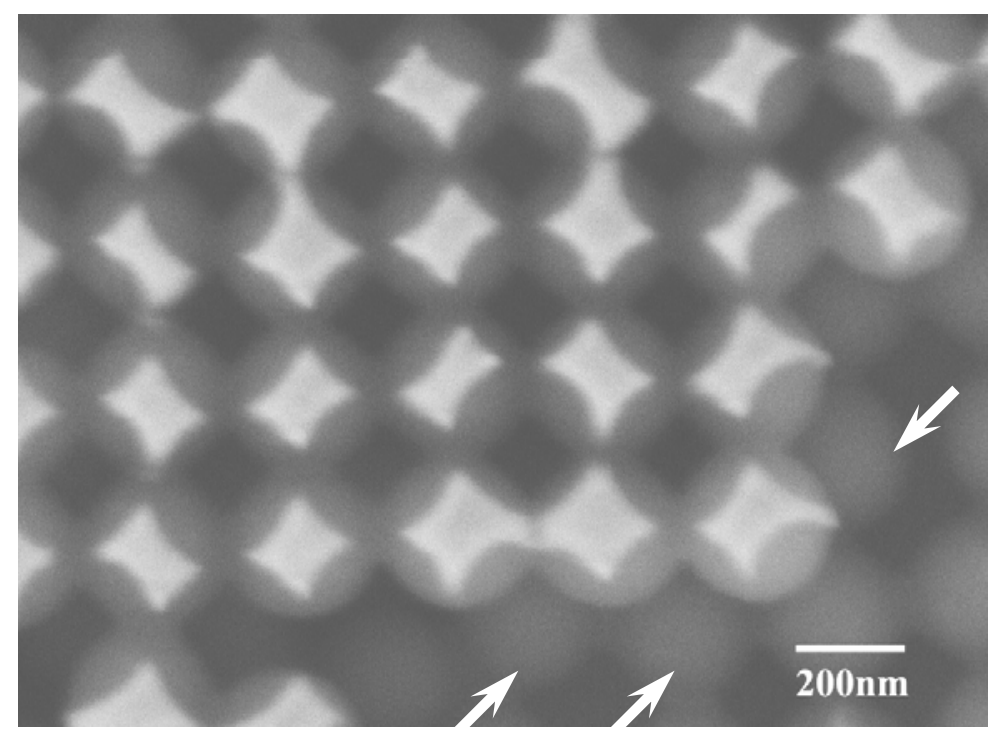

Figure S1 SEM picture of $270 \mathrm{~nm}$ polystyrene spheres with Au-patterned surfaces generated by templating colloidal crystals with (100) facets parallel to the substrates. As the stacking symmetry of the colloidal crystals used is "ABAB", in which the sphere of the third layer is located just beneath that of the first layer, no Au patterns were observed in the spheres in the third layer, indicated by arrows. 